\title{
Transition of originally external healthcare providers into local researchers: a case study of support activities in So-so District, Fukushima after the 2011 triple disaster
}

\author{
Akihiko Ozaki ${ }^{1,2, *}$, Toyoaki Sawano ${ }^{1,3,4}$ and Masaharu Tsubokura ${ }^{1,4}$ \\ ${ }^{1}$ Research Center for Community Health, Minamisoma Municipal General Hospital, 2-54-6 Takamicho, Haramachi, Minamisoma, \\ Fukushima, Japan. \\ ${ }^{2}$ Department of Breast Surgery, Jyoban Hospital of Tokiwa Foundation, Iwaki, Fukushima, Japan. \\ ${ }^{3}$ Department of Surgery, Jyoban Hospital of Tokiwa Foundation, Iwaki, Fukushima, Japan. \\ ${ }^{4}$ Department of Radiation Health Management, Fukushima Medical University School of Medicine, Fukushima, Fukushima, Japan.
}

Received: 14 October 2020 / Accepted: 11 November 2020

\begin{abstract}
There is a growing emphasis on the code of conduct in disaster research. It has recently been suggested that the involvement of local researchers is essential to achieving ethical behaviors when external researchers conduct fieldwork and study disaster-affected areas so that they can reflect local voices in their process and outputs. However, it is not always possible to fulfill these conditions due to the nature and preexisting resources of the affected area. Following Japan's 2011 triple disaster, a team of healthcare providers came in for support activities in the northern coastal area of Fukushima Prefecture. They primarily worked as clinicians before going beyond their original roles and eventually functioning as local researchers by drawing on the commitment of local stakeholders both inside and outside of medical institutions. This case study conducted an in-depth analysis on the initiatives and networks of originally external healthcare providers and discussed essential elements to working as local researchers following an unprecedented disaster with complex and persistent health, social, and political impacts. In conclusion, this has contributed to the development of evidence related to radiation protection and other health issues and supports the need for ethical behavior in disaster research.
\end{abstract}

Keywords: ethics in disaster research / healthcare providers / local population / So-so District / Japan's triple disaster

\section{Introduction}

A disaster can be defined as "an event that harms or kills a significant number or people or otherwise severely impairs or interrupts their daily lives in civil society" (Zack, 2009). Disasters have continued to be a major threat to human health and well-being (Kishore et al., 2018; Shultz et al., 2020), and have been increasing in frequency and intensity due to climate change (i.e., floods, hurricanes, and bushfires) (Forzieri et al., 2017). In addition, the risk of man-made disasters, namely nuclear disasters, may be increasing due to the continuous construction of nuclear reactors globally (World Nuclear Association, 2020). Naturally, there has been a pressing need to estimate the health impacts of disasters and develop effective countermeasures against future events based on gained knowledge and past experiences. Currently, the importance of disaster research is more heightened at present.

*Corresponding author: aozaki-tky@umin.ac.jp
In accordance with actual research conducted in disaster areas, awareness of the importance of disaster ethics has been increasing (Gaillard and Peek, 2019). Traditionally, disaster ethics has primarily underlined the fair and equal allocation of limited resources to save as many human lives in disasters as possible (Zack, 2009; Davis, 2014; Leider et al., 2017). However, as in other disciplines (Guillemin and Gillam, 2004), the emphasis on the code of conduct among researchers, who perform field work and research in disaster-affected areas, has been growing (Gaillard and Peek, 2019, 2020) primarily because scholars and societies have been gradually becoming more aware of the unique nature of disaster research. Disaster research tends to cause tensions in disaster victims and personnel engaged in recovery work, since these studies often approach physically and emotionally distressed people immediately after the aftermath of disasters (Gaillard and Peek, 2019). As such, poor awareness of the proper code of conduct not only causes distrust in disaster research in victims and those working for recovery, but also hinders the sound development of evidence bodies for diverse disaster impacts. 
Therefore, it is extremely important to ensure that all disaster research is ethically conducted.

In this context, Gaillard and Peek (2020) suggest that external researchers who perform studies in disaster-affected areas should be mindful of three principles. First, research themes should be locally relevant, besides contributing to future disaster risk reduction. Second, local residents' voices should be emphasized in both research processes and outputs. Third, local researchers should be involved in the entire research process. However, these principles may not always be feasible depending on the nature of the affected area. For example, in areas that have no academic institutions and no local researchers to work with, the third principle may not be achievable. Without the involvement of local researchers, the first and second principles may not be feasible either, because of the difficulty in building sound relationships with local residents. However, no pertinent solutions or alternative plans have been proposed to address this problem yet.

Japan's triple disaster in 2011 (earthquake, tsunami, and the Fukushima Daiichi Nuclear Power Plant [FDNPP] accident) was a multi-faceted disaster that caused several problems for the local communities in affected areas (The National Diet of Japan Fukushima Nuclear Accident Independent Investigation Commission, 2012). So-so District - the Northern coastal area of Fukushima Prefecturewas where the FDNPP was located, and this area was affected by all components of the triple disaster (Tsubokura, 2018; Zhang et al., 2014). On March 12, 2011, Japan's central government issued a mandatory evacuation order within the 20-kilometer radius of the FDNPP, and on March 15, 2011, the government additionally issued indoor sheltering orders within the 20-30-kilometer radius of the FDNPP (The National Diet of Japan Fukushima Nuclear Accident Independent Investigation Commission, 2012). These instructions were issued to safeguard local residents from exposure to the ionizing radiation, but health impacts associated with direct radiation exposure have been estimated to be negligible (United Nations Scientific Committee on the Effects of Atomic Radiation, 2014). However, it has been demonstrated that secondary health issues indirectly related to radiation exposure are diverse and have enormous health burdens (Tsubokura, 2018; Zhang et al., 2014). Long-term mass evacuation among the local residents and subsequent changes in demographics (e.g., population aging and depopulation) have caused social fluctuations in local communities (Morita et al., 2018; Zhang et al., 2014), leading to decreased informal care received at both household and community levels (Morita et al., 2016; Ozaki et al., 2016a, 2017a, 2017b). This has deteriorated social infrastructure, including health services in the affected areas (Ochi et al., 2016). Further, worsening lifestyle diseases (e.g. diabetes) and psychological stresses, including stigma against radiation exposure, have been reported (Nomura et al., 2016b; Sawano et al., 2018a). As such, while most evacuation orders have been lifted in the area around the FDNPP, the impacts of the disaster remain strong after nine years. Given the disaster's diverse long-term physical, psychological, and social impacts, conflicts can easily occur among researchers and local residents. In fact, in 2019, there was a massive controversy regarding the data collection process and handling of personal information on population-level external radiation exposure in a local municipality near So-so District (Sawano et al., 2019b).
In this context, one of the biggest challenges in So-so District following the 2011 triple disaster was the lack of academic institutions and local researchers. This may have been natural considering that nuclear power plants tend to be constructed in remote areas, but a lack of local academic institutions and researchers meant that there were no solid frameworks to connect local voices to external researchers when this massive disaster occurred in 2011. As such, healthcare providers (HCPs) who originally visited the area for support activities following the disaster and remained for clinical care and public health research in the long term may have played important roles in bridging these gaps.

During the past nine years following this triple disaster, our team of HCPs has primarily worked as clinicians in several medical institutions in So-so District. Simultaneously, we have participated in several public health activities, including an individual-level internal radiation exposure monitoring program. Moreover, we have analyzed and summarized diverse public health issues identified in the course of our clinical care, provided feedback to local residents and local municipality offices, and conveyed these findings globally by means of scientific publications and other media. Further, we sought help from external researchers to decipher the diverse health and social issues present after the disaster. However, information on the elements that made such transitions possible, particularly in the relationships made with local stakeholders, which represents a key aspect of ethical behavior in disaster research is lacking.

This work presents our initiatives in So-so District in the nine years following the 2011 Japan's triple disaster, and aims to lay out the qualities required for an external HCPs to function as locals researchers. The stakeholders' roles were extensively described to analyze their relationship with HCPs. We believe that an in depth analysis of our initiatives would provide important lessons in the aftermath of a disaster, and help establish a cooperative relationship with local stakeholders and other residents. We also discuss the generalizability of the research to other disaster situations.

\section{Case analyses}

We present a total of three cases that illustrate how originally external HCPs can work as local researchers who investigate local health issues and act as hubs for external researchers. We describe the first two cases as examples of the former role and the last case as an example of the latter role.

\subsection{Case 1: individual-level internal radiation exposure monitoring}

In So-so District, several medical institutions initiated individual-level internal radiation exposure monitoring following the 2011 triple disaster, and Minamisoma Municipal General Hospital (MMGH) - the largest municipality in the So-so District with a population of 70,919 as of March 1, 2011 (Morita et al., 2018) - was one of these medical institutions (Nomura et al., 2017). An early phase introduction process of whole body counter (WBC) units to the monitoring program in MMGH has been summarized elsewhere (Nomura et al., 2017). In short, in the first three months of the program (from 
July to September 2011), two chair-type WBC units (Anzai Medical Co., Ltd., Tokyo, Japan and Fuji Electric Co., Ltd., Tokyo, Japan) were utilized, which were replaced in late September 2011 by a standing-type WBC (FASTSCAN Model 2251, Canberra Inc., Meriden, CT, USA). While this program was originally initiated for local residents aged six years or older because of the nature of the devices (Nomura et al., 2017), there were massive concerns for radiation exposure of infants and small children among the local residents. This led to the development of BABYSCAN (Canberra Inc., Meriden, CT, USA), a WBC optimized for measuring internal radiation exposure in infants and small children (height $<130 \mathrm{~cm}$ ) (Hayano et al., 2014); its introduction to the MMGH in July 2014 enabled the monitoring of those aged younger than six years (Tsubokura et al., 2020).

This program was essentially a dedicated effort of the local municipality office of Minamisoma City and MMGH. Indeed, the municipality office and hospital staff of Minamisoma City and MMGH played a primary role in the program's operations. Additionally, one of the authors (MT) consistently supported this program as a radiation expert. When the disaster happened, he was among the numerous HCPs who visited So-so District for support activities as clinicians; he has since expanded his role beyond clinical care while continuing his support work. The role of the HCP has expanded to other areas beyond the internal radiation monitoring program at MMGH. His work can be largely categorized as evidence building of effective radiation protection after the nuclear disaster and communications with local residents.

Regarding evidence building of radiation protection, MT has worked on the health impacts of radiation exposure from various aspects. He worked on both internal and external radiation exposure (Tsubokura et al., 2015), including an initial report on internal radiation exposure after the 2011 triple disaster (Tsubokura et al., 2012); was committed to radiation monitoring programs in multiple areas inside and outside Soso District (Tsubokura et al., 2017; Akiyama et al., 2015; Hayano et al., 2013); analyzed several key populations for radiation exposure, including pregnant women and children (Yamamoto et al., 2019; Tsubokura et al., 2013); and conducted risk comparisons for health impacts induced by radiation exposure with other important secondary health issues (Murakami et al., 2015; Murakami et al., 2017). He has also worked on secondary health issues including diabetes and obesity using local population data (Nomura et al., 2016a, 2016b). His work has been cited in several key publications, such as the 2013 report by the United Nations Scientific Committee on the Effects of Atomic Radiation, and has become a foundation of scientific knowledge gained after the 2011 triple disaster (United Nations Scientific Committee on the Effects of Atomic Radiation, 2014).

Throughout this process, the commitment of local municipality offices and medical institutions were extraordinary. For example, data provided by Soma City and Minamisoma City became a foundation for MT and his colleagues to investigate health related issues related to radiation or otherwise. Further, the municipality office of Minamisoma City and MMGH launched Research Center for Community Health in the MMGH facility in 2018, with MT as its director and apportioning budgets for a continuous research program. Indeed, this decision reflected their awareness of the critical nature of long-term and diverse health issues arising from the 2011 triple disaster. Several private medical institutions, including Hirata Central Hospital and Tokiwa Foundation, also operated monitoring programs for internal radiation exposure at the individual level. In this respect, local municipalities and medical institutions played an important role in safeguarding the health of local residents and in collecting related evidence after the disaster.

Regarding communications with local residents, MT performed multiple activities. First, he provided face-to-face feedback about the findings of the monitoring program. In BABYSCAN, he and his staff held counseling sessions with the examinees and their guardians at MMGH (Tsubokura et al., 2020), where he not only directly conveyed the findings of the monitoring but also explained the findings' interpretation and implications in their daily lives (Tsubokura et al., 2020), while carefully listening to their concerns and giving concrete advice to mitigate them (Tsubokura et al., 2020). Second, MT repeatedly held small workshops for radiation education and communication that targeted local residents, particularly during the initial years after the disasters. In these workshops, residents could freely talk about their experiences and concerns and ask questions to other participants and workshop hosts. Third, MT also conducted lectures on radiation education for elementary, junior high, and high school students to indulge requests from local teachers and authorities. Since younger generations are more likely to suffer from the stigma of potential radiation exposure in the long term (Sawano et al., 2018a), these educational lectures were conducted to help them regain their sense of safety and self-confidence.

A notable local stakeholder during these initiatives was Veteran Mothers' Society, a nonprofit organization (NPO) which included local mothers in Minamisoma City. During sessions with local residents, the Veteran Mother's Society used its strong network to bring together participants for disseminating local data to those who urgently required them. Key contents of the workshops were summarized as "Dr. Tsubokura's Radiation Lecture", and its Japanese and English versions were published in August and October 2014. As of September 2020, approximately 50,000 and 10,000 copies of Japanese and English booklets have been freely distributed by the NPO.

In summary, beyond the original role of a clinician, MT has also worked as a local researcher for radiation protection. Abeysinghe et al. (2020) reported that the roles of HCPs extended beyond their primary roles as clinicians after the 2011 triple disaster, and become more proactive with the general public. This case further reiterates their statement that the efforts of local and other stakeholders became a foundation of such activities of HCPs. We believe that this is a critical point in how originally external HCPs could serve as local researchers who investigate locally relevant health issues occurring after a disaster.

\subsection{Case 2: summarization and publications of clinical cases encountered in daily clinical care}

A critical aspect of disaster research is to recognize that every disaster is a unique phenomenon with distinct features. Indeed, various elements can affect the damage and recovery 
process of disasters, including the type and scale of the disasters, the availability of medical and other resources, historical, societal, and political backgrounds of affected communities. This was particularly true in So-so District after the 2011 triple disaster, as evidenced by the diverse impacts on the area. Consequently, it would have been difficult to summarize health-related and other issues in So-so District without field work and/or support activities. This is important given that sensational academic articles that merely analyze large databases without reflecting local situations of disasteraffected areas have been repeatedly published following the triple disaster (Kobashi et al., 2020).

In this context, the strength of HCPs is their primary role of examining health concerns of patients. Following MT, several external HCPs entered So-so District, including AO and TS, the remaining authors of this work. They entered as full-time physicians in 2014, and like MT, primarily worked as clinicians in the area besides being local researchers. As local researchers, their main research topics involved investigating secondary health issues of the disaster. When they encountered unique cases in daily practice, they not only treated them but also documented them-mainly as case reports. While the evidence level of these case reports may have been low, it should be noted that a publication of case reports is an essential element of clinical practice in a disaster-affected area. This primarily worked as an effective feedback tool for concerned patients and a communication catalyst with other local residents. Additionally, this shed light on underrepresented but important existing problems in the aftermath process and helped HCPs develop research hypotheses to be evaluated for subsequent larger-scale studies. Using this bottom-up approach, we worked on several key health issues, as described hereafter.

One of AO's several research themes involved insect and animal injuries. The key characteristics of the triple disaster were the large-scale mandatory evacuation, and thus abandonment of residential areas (Zhang et al., 2014). From 2015 to 2016, he experienced multiple cases of insect and animal injuries in an outpatient MMGH office. Among them, he summarized a decontamination worker with a snakebite in a case report (Ozaki et al., 2016c). This eventually led him to hypothesize that the abandonment of these areas may have expanded the numbers and habitats of these animals, particularly in an evacuation zone, and that the progress of restoration work would naturally increase the encounters between these species and humans (Ozaki et al., 2016b). He summarized and published an observational study regarding an increased number of hospital visits due to hymenopteran stings from 2015 to 2016 (Ozaki et al., 2017d). This is a typical example of a bottom-up approach in which clinical care led to the development of a research theme.

AO has also worked on several case reports (Ozaki et al., 2016a, 2017a, 2017c) and observational analyses (Ozaki et al., 2017b) to explore the health impacts among cancer patients after the triple disaster, mainly targeting breast cancer patients. Notably, this work was possible because of a local $\mathrm{HCP}$ - OA's boss at the MMGH - who continuously treated breast cancer patients during the 2011 triple disaster with only a few months of interruption immediately after the disaster (Ozaki et al., 2020b). AO summarized data obtained from medical records of his patients.
Similarly, TS also employed a bottom-up approach for key health issues following the 2011 triple disaster such as the health of decontamination workers (Sawano et al., 2016a, 2016b, 2018a, 2020). Further, he covered some radiological issues using this approach (Sawano et al., 2019a). Needless to say, the clinical care of these patients preceded the publication of their case reports. In addition, he involved a local stakeholder to improve the health of decontamination and restoration workers. He held multiple seminars for the improvement of awareness of lifestyle diseases in cooperation with local construction companies that hired them. Other than $\mathrm{AO}$ and TS, similar examples of bottom-up approach have been described by HCPs who entered the area following the same disaster (Hori, submitted; Morita et al., 2015), drawing important lessons for another nuclear accident in the future.

In summary, a bottom-up approach may be advantageous in complex disasters such as the 2011 triple disaster because this defines an interaction with the patients as the starting point of research. The presence of local stakeholders including local HCPs was a critical component of this approach, which could lead to interventions aimed at improving the health status of the concerned population. Nonetheless, this approach is not a panacea and has several limitations due to its small sample size and drawbacks in data synthesis. In the following case, we will describe how we expanded our network with external researchers.

\subsection{Case 3: networking with external researchers}

We have described how, as local researchers, HCPs conducted locally relevant research after the 2011 triple disaster with various local stakeholders. While this approach has its own strengths, it is often difficult to fully assess the health impacts of the disaster relying solely on the skills of HCPs. Naturally, since the beginning of his work, MT has always been keen to collaborate with external researchers. In particular, a component of the nuclear disaster urged him to collaborate with physicists. However, in this section, we focus on two unique collaborations with external researchers that occurred during our study.

First is the collaboration with specialists in quantitative risk assessment, which identifies possible hazard or threats, analyzes their causes and consequences, and describes their risks (Aven, 2011). Thereby, it enables a direct comparison of different risks (Aven, 2011). While the focus was on health effects of direct radiation exposure following the triple disaster, it was gradually observed that mass evacuation and non-communicable diseases were also primary contributors to health problems. However, there had been no previous framework to compare the scales of these two health risks. Thus, MT, collaborating with specialists in quantitative risk assessment, published several articles, such as on a comparison of the health risks between evacuation and radiation and between diabetes mellites and radiation (Murakami et al., 2015, 2017).

The second example involves social scientists. Collaboration with researchers at the University of Edinburgh has provided another dimension to describe how the disaster impacted local health systems in So-so District. Specifically, we published multiple qualitative studies on medical and other 
essentials and HCPs' roles based on semi-structured interviews with local HCPs who actually experienced the disaster (Abeysinghe et al., 2017, 2020). This collaboration has further extended to a network entitled "Health, Risk, Disaster (HeaRD): Social Science and Post-Disaster Community Reconstruction" with the support of the Economic and Social Research Council in the United Kingdom in 2019 (Ozaki et al., 2020a) to comprehensively capture the health and social impacts of the triple disaster.

The HeaRD has been keen to listen to local voices. We held a symposium at MMGH and Fukushima Medical University under this network in 2019, where we invited local stakeholders in So-so District as speakers, including a high school teacher who educated students on the effects of radiation, and a local construction company president who led restoration work in the aftermath of the 2011 triple disaster. We believe that these gatherings which involve local residents, local researchers, and external researchers are instrumental in developing locally relevant research ideas.

In summary, relationships with external researchers were originally initiated from the research questions that arose from our local activities and helped local researchers address their questions via scientific publications. In the meantime, these relationships may have also helped external researchers to perform locally relevant research, with local researchers working as hubs to connect local voices to external researchers. In this regard, a bottom-up approach would encourage ethical behavior in disaster research.

\section{Discussion}

This report presents three concrete cases of the activities we conducted in So-so District following the 2011 triple disaster, which demonstrate that commitments by local stakeholders were key components for us to work as local researchers in the affected area. Specifically, in our case, stakeholders in So-so District were the local NPO, staff at medical institutions, and staff at municipality offices. As this does not occur naturally, we proactively sought their cooperation for our activities by building trust. As such, we believe that external HCPs need to possess three attributes.

First, respect and sympathy towards local residents is required. The 2011 triple disaster inflicted substantial damage on So-so District. For example, in Minamisoma City, more than a thousand residents were killed and more than one hundred individuals were reported missing after the tsunami. Naturally, numerous residents lost their family members, relatives and friends and were left homeless or jobless. While their experiences could be a source of data for research, they should be sought only after sound relationships were established with them. Rather, priority should be placed to the delivery of care and/or support to these individuals. In this context, the biggest strength of HCPs is their ability to see patients. As for local residents, healthcare is an essential support that is needed following disasters. We believe that the maximization of this skill helped us gain the trust of local residents and establish ourselves as local researchers in our case.

Second, long-term commitment is essential. The former Governor of Ache Indonesia, Irwandi Yusuf, criticized
Western external researchers following the 2004 tsunami and 2005 armed conflict as "guerrillas applying hit-and-run tactics" (Missbach, 2011). This means that these researchers made a quick search for relevant investigations and quickly left to write, publish, and make their careers elsewhere, without sharing their findings with local researchers and residents (Missbach, 2011). This criticism can be applicable to HCPs that came into the area affected by the disaster and did research unless they accept the necessary long-term commitment.

Indeed, we believe that HCPs have an advantage in terms of long-term commitment to disaster-affected areas. For example, in So-so District, there have been no academic institutions to work with local public health issues; therefore, it may have been difficult for external researchers to find local positions that enable long-term fieldwork in the area. Instead, HCPs could be economically independent by committing to clinical care, though this has limited their amount of time and effort for fieldwork.

Third, diverse and favorable relationships with key stakeholders in external communities are important. In our case, besides external researchers, the presence of external supporters helped our activities. For example, the Foundation for Global Children - set up in 2010 as a grassroots private group to promote healthy growth through medical and educational support - assisted MT's initiative since the 2011 triple disaster. It set up a dormitory in Soma City for those who came to support the initiative in So-so District for no cost back in 2011, and continued running it until 2018. This also functioned as a critical catalyst to strengthen networks between local residents, our team, and external researchers. Such logistics were quite helpful particularly in the early phase when networks were relatively small and weak.

\subsection{Limitations}

This report has several limitations. First, we must disclose that we wrote this article from HCPs' perspectives and did not involve local stakeholders or other residents to evaluate our initiatives. Thus, our interpretations are somewhat arbitrary and do not reflect their views on our initiatives. Second, we only covered experiences of three HCPs who came into the area after the 2011 triple disaster, which limits the generalizability of findings. To gain more robust evidence on the attributes required for the external HCPs to work as local researchers, local stakeholders and other residents' views on our initiatives should be taken into account. Moreover, activities of other HCPs who visited the disaster-affected area should be explored. Further, given that every disaster is a unique phenomenon, the same approach may not be relevant with other disaster events. In this regard, it may be meaningful to seek those who work on similar initiatives in other major disasters globally.

Nevertheless, this was one of the first attempts to propose solutions to an increasing concern in ethical behavior in disaster research. This could be a framework that can function for evidence building even in the long term. This is a natural consequence given that ethical behavior has been increasingly regarded as an essential component of disaster research, though it is notable when comparing our activities with those following Chernobyl nuclear accident. In the aftermath of the 
Chernobyl nuclear accident, researchers visited the area to investigate those affected by the disaster only as research targets. Although this approach may have been successful in some aspects, it struggled to draw commitments from local stakeholders, and some important health and social issues now remain just as anecdotal evidence. In contrast, our activity highlighted the salience of the commitment of local stakeholders. As researchers, efforts should be made to offer evidence that can be verified by other researchers.

\section{Conclusions}

This report summarized our initiatives in the aftermath of the 2011 triple disaster. We primarily found that commitment of local stakeholders and other residents helped us function as local researchers. This has not only helped us proceed with our investigations ethically, but also enabled the development of evidence related to radiation protection and other health issues in the long term. While the generalizability of our case to other HCPs and other disaster events should be carefully evaluated, we believe that this bottom-up approach that underlines local issues and voices as a starting point of research could be a solution to the increasing concerns about ethical behavior in disaster research.

\section{References}

Abeysinghe S, Leppold C, Ozaki A, Morita M. 2020. Risk, uncertainty and medical practice: changes in the medical professions following disaster. Evid. Policy J. Res. Debate Pract. 16: $285-303$.

Abeysinghe S, Leppold C, Ozaki A, Morita M, Tsubokura M. 2017. Disappearing everyday materials: The displacement of medical resources following disaster in Fukushima, Japan. Soc. Sci. Med. 191: 117-124.

Akiyama J, Kato S, Tsubokura M, Mori J, Tanimoto T, Abe K, Sakai S, Hayano R, Tokiwa M, Shimmura H. 2015. Minimal internal radiation exposure in residents living south of the Fukushima Daiichi nuclear power plant disaster. PLoS One 10: e0140482.

Aven T. 2011. Quantitative risk assessment: the scientific platform. Cambridge: Cambridge University Press.

Davis AJ. 2014. Ethics needed for disasters: before, during, and after. Health Emerg. Disaster Nurs. 1: 11-18.

Forzieri G, Cescatti AE, Silva FB, Feyen L. 2017. Increasing risk over time of weather-related hazards to the European population: a data-driven prognostic study. Lancet Planet. Health 1: e200-e208.

Gaillard JC, Peek L. 2019. Disaster-zone research needs a code of conduct. Nature 575: 440-442.

Gaillard JC, Peek L. 2020. Towards a code of conduct for ethical postdisaster research. https://ahrecs.com/human-research-ethics/to wards-a-code-of-conduct-for-ethical-post-disaster-research (accessed September 28 2020).

Guillemin M, Gillam L. 2004. Ethics, reflexivity, and "ethically important moments" in research. Qual. Inq. 10: 261-280.

Hayano RS, Tsubokura M, Miyazaki M, Satou H, Sato K, Masaki S, Sakuma Y. 2013. Internal radiocesium contamination of adults and children in Fukushima 7 to 20 months after the Fukushima NPP accident as measured by extensive whole-body-counter surveys. Proc. Jpn. Acad. Ser. B Phys. Biol. Sci. 89: 157-163.
Hayano RS, Yamanaka S, Bronson FL, Oginni B, Muramatsu I. 2014. BABYSCAN: a whole body counter for small children in Fukushima. J. Radiol. Prot. 34: 645-653.

Hori A. 2020. Coming to terms with disaster-related trauma and earlier trauma by constructing a new identity: a case report. Radioprotection (submitted).

Kishore N, Marques D, Mahmud A, Kiang MV, Rodriguez I, Fuller A, Ebner P, Sorensen C, Racy F, Lemery J, Maas L, Leaning J, Irizarry RA, Balsari S, Buckee CO. 2018. Mortality in Puerto Rico after Hurricane Maria. N. Engl. J. Med. 379: 162-170.

Kobashi Y, Sawano T, Ozaki A, Tsubokura M. 2020. Misunderstanding of the purpose and intermediate results of the Fukushima health management survey could impede progress in the radiation protection field. J. Radiol. Prot. 40: 924-927.

Leider JP, Debruin D, Reynolds N, Koch A, Seaberg J. 2017. Ethical guidance for disaster response, specifically around crisis standards of care: a systematic review. Am. J. Public Health 107: e1-e9.

Missbach A. 2011. Ransacking the field? Crit. Asian Stud. 43: 373 398.

Morita T, Leppold C, Tsubokura M, Nemoto T, Kanazawa Y. 2016. The increase in long-term care public expenditure following the 2011 Fukushima nuclear disaster. J. Epidemiol. Community Health 70: 738 .

Morita T, Nomura S, Furutani T, Leppold C, Tsubokura M, Ozaki A, Ochi S, Kami M, Kato S, Oikawa T. 2018. Demographic transition and factors associated with remaining in place after the 2011 Fukushima nuclear disaster and related evacuation orders. PLoS One 13: e0194134

Morita T, Tanimoto T, Hori A, Kanazawa Y. 2015. Alcohol use disorder due to social isolation after a nuclear disaster in Fukushima. BMJ Case Rep. 2015.

Murakami M, Ono K, Tsubokura M, Nomura S, Oikawa T, Oka T, Kami M, Oki T. 2015. Was the risk from nursing-home evacuation after the Fukushima accident higher than the radiation risk? PLoS One 10: e0137906.

Murakami M, Tsubokura M, Ono K, Nomura S, Oikawa T. 2017. Additional risk of diabetes exceeds the increased risk of cancer caused by radiation exposure after the Fukushima disaster. PLoS One 12: e 0185259 .

Nomura S, Blangiardo M, Tsubokura M, Ochi S, Hodgson S. 2016a. School restrictions on outdoor activities and weight status in adolescent children after Japan's 2011 Fukushima Nuclear Power Plant disaster: a mid-term to long-term retrospective analysis. BMJ Open 6: e013145.

Nomura S, Blangiardo M, Tsubokura M, Ozaki A, Morita T, Hodgson S. 2016b. Postnuclear disaster evacuation and chronic health in adults in Fukushima, Japan: a long-term retrospective analysis. BMJ Open 6: e010080.

Nomura S, Tsubokura M, Murakami M, Ono K, Nishikawa Y, Oikawa T. 2017. Towards a long-term strategy for voluntary-based internal radiation contamination monitoring: representativeness of the monitoring results in Fukushima, Japan. Int. J. Environ. Res. Public Health 14.

Ochi S, Tsubokura M, Kato S, Iwamoto S, Ogata S, Morita T, Hori A, Oikawa T, Kikuchi A, Watanabe Z, Kanazawa Y, Kumakawa H, Kuma Y, Kumakura T, Inomata Y, Kami M, Shineha R, Saito Y. 2016. Hospital staff shortage after the 2011 triple disaster in Fukushima, Japan - An earthquake, tsunamis, and nuclear power plant accident: a case of the Soso District. PLoS One 11: e0164952.

Ozaki A, Elstow L, Murakami M, Tsubokura M, Abeysinghe S. 2020a. Lessons learned from Fukushima, Japan: in what ways can 
the social sciences help to mitigate some of the health impacts of disaster? QJM 113: 237-238.

Ozaki A, Leppold C, Sawano T, Tsubokura M, Tsukada M, Tanimoto T, Kami M, Ohira H. 2017a. Social isolation and cancer management - advanced rectal cancer with patient delay following the 2011 triple disaster in Fukushima, Japan: a case report. J. Med. Case Rep. 11: 138.

Ozaki A, Leppold C, Tsubokura M, Tanimoto T, Saji S, Kato S, Kami M, Tsukada M, Ohira H. 2016a. Social isolation and cancer management after the 2011 triple disaster in Fukushima, Japan: a case report of breast cancer with patient and provider delay. Medicine (Baltimore) 95: e4027.

Ozaki A, Nomura S, Leppold C, Tsubokura M, Sawano T, Tsukada M, Morita T, Tanimoto T, Saji S, Kato S, Yamaoka K, Nakata Y, Ohira H. 2020b. Breast cancer provider interval length in Fukushima, Japan, after the 2011 triple disaster: a long-term retrospective study. Clin. Breast Cancer 20: e127-e150.

Ozaki A, Nomura S, Leppold C, Tsubokura M, Tanimoto T, Yokota T, Saji S, Sawano T, Tsukada M, Morita T, Ochi S, Kato S, Kami M, Nemoto T, Kanazawa Y, Ohira H. 2017b. Breast cancer patient delay in Fukushima, Japan following the 2011 triple disaster: a long-term retrospective study. BMC Cancer 17: 423.

Ozaki A, Sawano T, Tsukada M, Ohira H, Leppold C, Tanimoto T. 2016b. Abandoned areas in post-disaster Fukushima, Japan. QJM 109: 353-354.

Ozaki A, Tanimoto T, Leppold C, Tsubokura M, Kato S, Tsukada M, Kami M, Ohira H. 2016c. A possible association between the resumption of agricultural activities and a venomous snakebite after Fukushima nuclear crisis. Oxf. Med. Case Rep. 2016: 22-23.

Ozaki A, Tsubokura M, Leppold C, Sawano T, Tsukada M, Nemoto T, Kosugi K, Nishikawa Y, Kato S, Ohira H. 2017c. The importance of family caregiving to achieving palliative care at home: a case report of end-of-life breast cancer in an area struck by the 2011 Fukushima nuclear crisis: a case report. Medicine (Baltimore) 96: e8721.

Ozaki A, Yokota T, Nomura S, Tsubokura M, Leppold C, Tanimoto T, Miura T, Yamamoto K, Sawano T, Tsukada M, Kami M, Kanazawa Y, Ohira H. 2017d. Decontamination work and the long-term increase in hospital visits for hymenoptera stings following the Fukushima nuclear disaster. Disaster Med. Public Health Prep. 11: 545-551.

Sawano T, Kambe T, Seno Y, Konoe R, Nishikawa Y, Ozaki A, Shimada Y, Morita T, Saito H, Tsubokura M. 2019a. High internal radiation exposure associated with low socio-economic status six years after the Fukushima nuclear disaster: a case report. Medicine (Baltimore) 98: e17989.

Sawano T, Nishikawa Y, Ozaki A, Leppold C, Tsubokura M. 2018a. The Fukushima Daiichi Nuclear Power Plant accident and school bullying of affected children and adolescents: the need for continuous radiation education. J. Radiat. Res. 59: 381-384.

Sawano T, Takita M, Senoo Y, Nishikawa Y, Crump A, Tsubokura M. 2019b. The responsibility of the Japanese Media, The Fukushima accident \& the use of personal data for research. QJM.

Sawano T, Tanaka H, Watanabe D, Ozaki A, Tsukada M, Nishikawa Y, Saito H, Shimada Y, Morita T, Ohira H, Tsubokura M. 2020. Concealment of trauma and occupational accidents among Fukushima nuclear disaster decontamination workers: a case report. J. Occup. Health 62: e12123.

Sawano T, Tsubokura M, Leppold C, Ozaki A, Fujioka S, Nemoto T, Kato S, Oikawa T, Kanazawa Y. 2016a. Klebsiella pneumoniae sepsis deteriorated by uncontrolled underlying disease in a decontamination worker in Fukushima, Japan. J. Occup. Health 58: $320-322$.
Sawano T, Tsubokura M, Ozaki A, Leppold C, Kato S, Kambe T. 2018b. Legionnaires' disease as an occupational risk related to decontamination work after the Fukushima nuclear disaster: a case report. J. Occup. Health 60: 527-528.

Sawano T, Tsubokura M, Ozaki A, Leppold C, Nomura S, Shimada Y, Ochi S, Tsukada M, Nemoto T, Kato S, Kanazawa Y, Ohira H. 2016b. Non-communicable diseases in decontamination workers in areas affected by the Fukushima nuclear disaster: a retrospective observational study. BMJ Open 6: e013885.

Shultz JM, Sands DE, Kossin JP, Galea S. 2020. Double environmental injustice - climate change, hurricane Dorian, and the Bahamas. N. Engl. J. Med. 382: 1-3.

The National Diet of Japan Fukushima Nuclear Accident Independent Investigation Commission. 2012. Overview of the damage and how it spread. The official report of the Fukushima Nuclear Accident Independent Investigation Commission. https://warp.da. ndl.go.jp/info:ndljp/pid/3856371/naiic.go.jp/wp-content/uploads/ 2012/08/NAIIC_Eng_Chapter4_web.pdf (accessed September 28 2020).

Tsubokura M. 2018. Secondary health issues associated with the Fukushima Daiichi nuclear accident, based on the experiences of Soma and Minamisoma Cities. J. Natl. Inst. Public Health 67: 7183.

Tsubokura M, Gilmour S, Takahashi K, Oikawa T, Kanazawa Y. 2012. Internal radiation exposure after the Fukushima nuclear power plant disaster. JAMA 308: 669-670.

Tsubokura M, Kato S, Morita T, Nomura S, Kami M, Sakaihara K, Hanai T, Oikawa T, Kanazawa Y. 2015. Assessment of the annual additional effective doses amongst Minamisoma children during the second year after the Fukushima Daiichi nuclear power plant disaster. PLoS One 10: e0129114.

Tsubokura M, Murakami M, Nomura S, Morita T, Nishikawa Y, Leppold C, Kato S, Kami M. 2017. Individual external doses below the lowest reference level of $1 \mathrm{mSv}$ per year five years after the 2011 Fukushima nuclear accident among all children in Soma City, Fukushima: a retrospective observational study. PLoS One 12: e0172305.

Tsubokura M, Nabeshima Y, Murakami M, Nemoto T, Kambe T, Nonaka S, Shimada Y, Kobashi Y, Ozaki A, Oikawa T. 2020. Usefulness of the whole-body counter for infants and small children (BABYSCAN) as a risk communication tool after the Fukushima Daiichi nuclear power plant incident. Proc. Jpn. Acad. Ser. $B$ 96: 70-78.

Tsubokura M, Shibuya K, Kato S, Oikawa T, Kanazawa Y. 2013. Acute intake of radionuclides immediately after the incident as the main contributor of the internal radiation exposure after Fukushima Daiichi nuclear disaster. JAMA Pediatr. 167: 11691170 .

United Nations Scientific Committee on the Effects of Atomic Radiation. 2014. Annex a: levels and effects of radiation exposure due to the nuclear accident after the 2011 great east-Japan earthquake and tsunami. New York: United Nations Scientific Committee on the Effects of Atomic Radiation.

World Nuclear Association. 2020. Plans for New Reactors Worldwide. https://www.world-nuclear.org/information-library/ current-and-future-generation/plans-for-new-reactors-worldwide. aspx (accessed September 28 2020).

Yamamoto K, Nomura S, Tsubokura M, Murakami M, Ozaki A, Leppold C, Sawano T, Takita M, Kato S, Kanazawa Y, Anbe H. 2019. Internal exposure risk due to radiocesium and the consuming behaviour of local foodstuffs among pregnant women in Minamisoma City near the Fukushima nuclear power plant: a retrospective observational study. BMJ Open 9: e023654. 
Zack N. 2009. Ethics for disaster. Maryland: Rowman \& Littlefield Publishers.

Zhang H, Yan W, Oba A, Zhang W. 2014. Radiation-driven migration: the case of Minamisoma City, Fukushima, Japan, after the
Fukushima nuclear accident. Int. J. Environ. Res. Public Health 11: 9286-9305.

Cite this article as: Ozaki A, Sawano T, Tsubokura M. 2020. Transition of originally external healthcare providers into local researchers: a case study of support activities in So-so District, Fukushima after the 2011 triple disaster. Radioprotection 55(4): 263-270 